Collaborating to Bring the Credibility Revolution to Giftedness and Talent Development Research

\begin{abstract}
Students have numerous opportunities to learn outside the classroom. However, with great choice comes great variability of both quality and of intent. To evaluate the effectiveness of outof-school programs generally - as well as individual programs specifically-we must know their intended effects (program goals) as well as their actual effects (program outcomes). Despite numerous existing evaluations and even more numerous claims, many programs and evaluations lack strong empirical support, suffer from biases, and are ripe for perverse incentives. In this paper, we propose greater adoption of large-scale collaborative research to provide more precise and accurate information about: effects of participation; heterogeneity of effects across contexts or demographic groups; resolve disagreements; and evolve to exploring new questions with greater confidence in previous findings. Implementing large-scale collaborative research practices that have helped catalyze a credibility revolution in psychology will help stakeholders make more informed decisions.
\end{abstract}

Keywords: Credibility Revolution, Giftedness, Open Science, Talent Development 
Collaborating to Bring the Credibility Revolution to Giftedness and Talent Development Research

Advanced learning contexts for gifted and talented students are exceptionally diverse. How students are identified, the age they are identified, how they are served, what else they do with their time, and a host of other relevant factors can vary across programs/contexts. This variability in contexts can have its benefits, but there are also costs. It creates a smorgasbord of opportunities for students, but also a smorgasbord of empirical findings that may or may not generalize to other programs or other contexts (i.e., if one program relies on an entirely different service model from another, data on program effectiveness does not automatically generalize from one program to the other). Such variety makes the evaluation of specific programs or even specific components of programs - much less broad generalization about "what works" — quite difficult. For example, although increasing test scores is a goal of test preparation programs, many gifted programs focus less on increasing math or language arts achievement test scores and more on constructs such as critical thinking or creativity (https://ncrge.uconn.edu/key-findings/). Heterogeneity of program goals should drive heterogeneity of program offerings, thus making a smorgasbord of opportunities appropriate from a program offering perspective.

These constraints on generality also create constraints on our knowledge of "what works." Such ignorance about "what works" (and what doesn't) prevents the field from improving its ability to provide effective learning contexts, which hinders the development of talent and the closure of achievement and excellence gaps. This is because flexibility allows programs to offer unique opportunities, but that very uniqueness limits knowledge of program effectiveness. If “the experts” don't know what works, how can parents looking to send their children to such programs or practitioners who may (or may not) want to make recommendations 
make informed decisions? Participating in a program may be a significant financial investment for families who want to know that their child will benefit. Even if a program has no financial costs, it still has an opportunity cost for a child participating in that program instead of doing something else.

The opportunity costs may be experienced asymmetrically across populations. For example, in the United States, parents from higher income levels spend far more on their children than do parents from low or even average-income income levels (Kornrich, 2016; Schneider, Hastings, \& LaBriola, 2018). For example, in 2000, parents in the highest income decile spent approximately $\$ 7,000$ per child per year compared to less than $\$ 1,000$ per year per child for families in the lowest decile (Kornrich, 2016). Such gaps in parental spending have been growing for decades. Whereas in 1980, the gap between the highest and lowest deciles was approximately $\$ 2,500(\$ 3600$ - $\$ 1,100)$, in 2010 the gap was approximately $\$ 6,700(\$ 7,500$ \$800). Since the 1990s, the largest part of the gap has constituted early childhood education and childcare - a component of out of school learning opportunity. The second-largest component was enrichment goods and activities. Such parental spending gaps highlight two important points. First, at least in the U.S., there has been significant growth in parental spending on both formal pre-school education as well as out-of-school enrichment activities for all groups and income levels. Second, access to and affordability of such programs is not evenly distributed. Families with fewer economic resources cannot afford to spend as much money on out-of-school programming as those with more resources. Related, the growth in spending by upper-income parents has far outstripped that of average- or lower-income parents. As such, those with resources can often afford to invest in superfluous interventions, but those with limited resources 
must be selective. The more accurate information that the research and evaluation community can provide, the more informed choices all stakeholders can make.

\section{Nullius in Verba}

Just as programs do not always deliver intended experiences and outcomes, the research community does not always live up to its aspirations. The oldest scientific society in the world is the Royal Society of England. Their motto, "nullius in verba" states that "on no one's word" should we believe anything. Instead, they require evidence. Modern research culture is built on such a perspective, but as has become clear in recent years, the edifice of evidence for many topics is often a house of cards. The numerous problems of the modern research apparatus have been summarized and explained elsewhere for education broadly (Makel, Smith, McBee, Peters, \& Miller, 2019; van der Zee \& Reich, 2018) as well as for gifted education specifically (Adelson \& Matthews, 2019; McBee, Makel, Peters, \& Matthews, 2018). Although not focused specifically on gifted and talented education, the problems identified in these papers likely generalize to out-of-school contexts. For example, writing about clinical trial research, Ioannidis (2005) claimed that "most published research findings are false" and that research findings were less likely to be true if they came from studies with smaller samples, reported smaller effect sizes, had a larger number of comparisons, and flexibility in design, definition, and outcomes, or if researchers were financially invested in the outcome. Although we have not conducted a formal analysis, we believe that such features are representative of much of the research on advanced learning and enrichment experiences for advanced learners, especially when compared to the clinical trial research that Ioannidis was criticizing.

Extrapolating from research from other fields, we believe several common flaws need fixing to increase confidence in — and precision of - research. Although extremely valuable, data 
are not divine. Data are less trustworthy when there are high rates of researchers using questionable research practices such as selectively reporting only studies that "worked", peeking at data to determine whether to collect more, excluding data after looking at its influence on results, and claiming to have predicted unexpected results (e.g., John, Lowenstein, \& Prelec, 2012). Similarly, when researchers attempt to replicate their own previous findings, their success rate tends to be higher than when independent research teams attempt to replicate those findings (Makel \& Plucker, 2014). And when those independent replication attempts rely on sufficient sample sizes, the resulting effects tend to be much smaller than the original results (Open Science Collaboration, 2015). Such biased reporting can occur due to selection of what outcomes are reported, the "spin" used on those outcomes, which studies are published, and which studies are cited (de Vries et al., 2018). Each stage of spin can make results appear far stronger and more consistent than the actual data support. These problems do not merge to form a solid research foundation that should be trusted by the public.

\section{Closing the Gap between Aspiration and Action}

Several initiatives have been developed across many scientific fields in reaction to the aforementioned problems. Vazire (2018) dubbed such actions as a credibility revolution in psychological research. At the heart of this credibility revolution was changing research behaviors to better align with scientific norms of universalism, communality, disinterestedness, and organized skepticism (Merton, 1942/1973). According to Vazire, to achieve this alignment, the standards by which research is evaluated must evolve to include factors such as transparency and openness, preregistration of hypotheses, the importance of independent direct replication of results, and a need for higher quality and quantity of evidence prior to making strong scientific claims. These actions fall under the umbrella term of open science (https://cos.io/). Recently, 
several research teams have argued that education (van der Zee \& Reich, 2018), special education (Adelson et al., 2019; Cook, Lloyd, Mellor, Nosek, \& Therrien, 2018), and gifted education research (McBee et al., 2018) could benefit from greater adoption of open science practices such as preregistration, data and materials sharing, preprints, badges, and registered reports (where literature reviews, proposed methods, and analytic plans undergo peer review prior to data collection to shift incentives from significance of results to significance of research questions and rigor of methods). Doing so would better align practice with the norms and values of scientific research, thereby leading to greater credibility.

\section{Problems with Giftedness Program Evaluation and Research}

Although greater adoption of open science practices would certainly increase the quality and credibility of research, we believe that there are some flaws relevant to research in gifted and talented contexts that require additional methodological improvement beyond adoption of open science practices. We will use out-of-school learning contexts as an example to illustrate our concerns. First, because out-of-school contexts in gifted education often have differing goals, identification criteria, program length, etc., it can be difficult to know whether findings from one program generalize to another. Even similar programs can have potentially meaningful differences from each other. For example, talent search programs such as the Duke University Talent Identification Program (TIP), Northwestern's Center for Talent Development (CTD), and Johns Hopkins Center for Talented Youth (CTY) are often considered similar to each other. With reason, they all rely on the same model developed by Julian Stanley. All three use above-level testing via a college admissions test (SAT or ACT) as a qualification mechanism and have similar program lengths and models. However, they use different qualifying scores for their programs and TIP does not offer course credit whereas CTD and CTY sometimes do. If course 
credit is salient for outcomes, these programs may differ from each other in some regard.

Knowing when and whether between-program differences influence relevant outcomes can help provide a more nuanced understanding of the effects of out-of-school programs.

A second concerns is related to that even when program goals are similar, they may still differ in how they identify students which can have an influence on what is considered effective or successful. For example, McBee and Makel (2019) showed how large variability in the number of students expected to be identified by particular definitions of giftedness even when relying on a single definition. Variability grows even greater when different definitions are considered. Even programs within a single state might operate differently despite being based on a single state-level definition of giftedness (they can offer different amounts of service or even the same service with varying levels of quality). Given this variability, assuming generalizability across who is identified and the effects of service across varying programs may not be appropriate.

Third, despite numerous existing evaluations and even more-numerous claims, many programs lack strong empirical support, suffer from biases, and are ripe for perverse incentives. The biases and perverse incentives come from program staff often being responsible for conducting evaluations of their own program. When such programs are evaluated by those responsible for running them, the possibility of confirmation bias becomes relevant. This is not because of ill-intent, but because people are human. This is not unique to gifted programs. For example, in education, research included in the U.S. Department of Education's What Works Clearinghouse is typically considered to be quite rigorous. However, a recent analysis found that when the evaluation was conducted by a group affiliated with the intervention, the typical effect size was over 1.5 times the typical effect size found when an evaluation was conducted by an 
independent team (Wolf, Morrison, Slavin, \& Risman, 2019). There may be several explanations for such a gap, but confirmation bias (inclination to seek and interpret results that align with existing beliefs) is likely among them.

When reviewing how to assess evidence in gifted education, Callahan and Moon (2007) split the types of evidence for gifted programs into speculative/anecdotal, theoretical, and empirical. They concluded that advice in the gifted education research literature goes from "armchair best thinking” (p. 305) to conflicting and hard-to-understand. They also noted that variability in factors such as program duration or staff training could lead to differences in results or program effectiveness, further contributing to potential limits on generalizability of published findings.

Fourth, studying the gifted necessarily results in small samples if research is limited to local populations of programs only evaluating their own interventions. To make statistical inferences, small samples are beyond useless; they are dangerous. They are dangerous because they can create a false veneer of rigor and sophistication (especially to stakeholders unfamiliar with statistics) that communicates more confidence than is merited. For example, the sample size needed to provide a result with sufficient precision to inform readers of something useful is often quite large (over 1,000), and larger than many published studies in gifted education use (Anderson, Kelley, \& Maxwell, 2017; Maxwell, Kelley, \& Rausch, 2008). However, samples of over 1,000 are not realistic for many gifted studies. Rather than adapt to meet rigor, researchers often ignore rigor to do what is convenient (e.g., using small convenience samples). Such behavior is cognitive dissonance personified. In addition to leading to the creation of results that cannot (and should not) be trusted, it undermines the credibility of the field by allowing published research findings to be easily ignored due to low quality. We believe that to best meet 
the needs of gifted and talented students, the field's research and evaluation efforts should be so rigorous that findings cannot be ignored. To accomplish this, the research and evaluation community needs to provide more informative results to be considered credible. We believe the road to such credibility is paved with large-scale collaborative research.

\section{Models of Collaborative Research}

The concept of large-scale collaborative research helping improve research quality has been introduced previously (Ioannidis, 2014; Uhlmann et al., 2018), including for education research (Makel et al., 2019), and is already regularly applied in fields like physics where papers can have thousands of authors (e.g., Aad et al., 2012; Abbot et al., 2017; Chatrchyan et al., 2012). Here, we focus specifically on how it can help better evaluate/understand learning contexts for gifted and talented students. Building on the work of Makel and colleagues (2019) who wrote about large-scale collaboration in education research broadly, in the following sections, we introduce five large-scale collaborative research types and discuss how they could be applied to learning contexts for gifted and talented students. When paired with greater adoption of open science practices, we believe they could better align research practices with scientific norms, reduce the prevalence of the problems listed above, and improve understanding of the effects of learning environments for gifted and talented students. This is not intended to be a methods brief that teaches readers how to conduct large-scale collaborations; that is beyond the scope of this manuscript. Our goal with this section is to introduce large-scale collaboration methods and outline how they could help improve upon existing research practices in gifted education.

\section{Participating Teams Evaluate Different Things}


In this form of collaboration, teams of researchers collaborate to select research projects to conduct, but then largely conduct individual research studies independently, coming back together to report their findings. In an effort to estimate replicability (collecting new data to assess previous results), other fields have established large-scale collaborations like this where participating groups evaluate previously published findings. At its most basic, this form of collaboration has collaborators evaluate different projects or programs in a coordinated manner. For example, in psychology, the Open Science Collaboration (OSC, 2015) conducted the Reproducibility Project: Psychology, assessing 100 previously-published psychology studies by following preregistered protocols as closely as possible with high statistical power. The group was able to successfully replicate 39 of the 100 original results. However, the magnitude of their findings were typically only about half as large as the original results.

Giftedness research could conduct similar evaluations of existing findings. For example, Rogers (2015) reported effects of a variety of acceleration practices. Each acceleration option had an associated mean academic effect, such as grade-skipping (0.67, based on 5 studies), talent search programs (0.34, based on 6 studies), and Saturday classes on a university campus (1.56, based on 1 study). But many of these studies were conducted decades ago, using varying qualification criteria, making generalizability to current programs tenuous. A large-scale collaborative research project could assess whether these results (and their corresponding magnitude) could be replicated today. Such updated precise information could be greatly informative for numerous stakeholders. Such a collaborative project might take the form of several university-based Saturday enrichment programs agreeing to replicate past research on such programs. They could pre-register their methods of evaluation, which would take into account the different program models and identification criteria. Applying such methods would 
inform all consumers of research (e.g., program owners as well as prospective participants) of expected results from participating in such a program and whether these results vary across implementation sites.

\section{Multi-Team Collaborations}

Large-scale collaborative research projects are not always focused on replicating previous findings; they can also collect new data on new ideas. In multi-team collaboration projects, multiple independent teams collaborate on a single project following an agreed-upon, preregistered protocol. Having multiple groups conduct the same study would provide better information on generalizability and replicability of the effect and its magnitude. Such collaborations require explicit research protocols beyond simply what data are collected, but also how they are collected, coded, analyzed, and interpreted; shared videos showing each of these steps may be helpful. The end results can then be interpreted individually (comparing results of specific research teams or specific sites) as well as together in a form similar to a meta-analysis. Multi-team collaborations can be particularly useful in gifted education when programs differ from each other (e.g., different qualifying scores, program length, goals, etc.). Understanding the effects of situational factors unique to particular programs can reveal which program components are (or are not) associated with effects. For example, gifted education researchers have long been interested in the effects of different types of grouping on academic achievement. Total School Cluster Grouping (Gentry, Paul, McIntosh, Fugate, \& Jen, 2014) or Schoolwide Cluster Grouping (Winebrenner, \& Brulles, 2008) involve narrowing the achievement levels within classrooms to better facilitate instructional targeting and differentiation. Advanced learners are grouped together in the same room to facilitate specialized instruction. The second-order meta-analysis by Steenbergen-Hu, Makel, and Olszewski-Kubilius 
(2016) found an overall effect size of $g=.33$ for within-class grouping (95\% CI [0.14, 0.52]), but with wide heterogeneity across studies $\left(I^{2}=65.98\right)$. This is of no surprise to anyone who has ever tried to implement cluster grouping. The schools where the intervention and research were conducted vary, the professional development and training provided to teachers varied, and the actual true effect varies. Applied to cluster grouping, a multi-team collaboration could take the form of several research teams agreeing on a set protocol for training, fidelity of implementation, data collection, and analysis. The teams would then implement the intervention and the results would be aggregated via traditional meta-analyses with covariates for the different settings. The benefit to such an approach is that findings are much more likely to capture the true effect of cluster grouping and reveal unbiased context-dependent variation than any single study conducted by one researcher or in one setting.

Organizations could independently conduct research on their own programs, but more informative knowledge would be generated if multiple organizations used a multi-team collaboration format. This is because such collaboration could reveal when/where heterogeneity exists, which would help to better reveal the effect of these out-of-school interventions than multiple independently run studies. Doing so would also greatly increase the available sample size, thereby allowing for the detection of smaller effects as well as sub-group analysis.

Although such collaborations require coordination, they provide far more knowledge than individual studies of a single intervention. As discussed above, multi-team collaborations are less likely to overestimate the size of the effect and are more likely to capture nuances to the effect itself. By assessing multiple programs, multi-team collaborations effectively control for extraneous factors to estimate the true effect and the conditions under which that effect appears. 
By having multiple collaborators and research sites, a single "study" conducted via multi-team collaboration can better understand what factors influence the effect and its magnitude.

\section{Persistent Collaboration}

Multi-team collaborations discussed above rely on finding partners for each

collaboration. Such work can be quite labor intensive, particularly for programs that do not have a research team. A form of collaboration that may be relevant in this situation is persistent collaboration. Persistent collaborations are a form of permanent infrastructure designed to create and foster such research collaborations. Consortia could be formed where individual researchers and programs could join and then choose to opt in or out of particular projects.

Such an infrastructure already exists within psychology, PsyAccelerator (https://psysciacc.org; Moshontz et al., 2018), and has over 500 participating research labs from 60 countries. A similar infrastructure could be created within gifted and talented field. For example, societies such as the National Association for Gifted Children or the European Council for High Ability could be the home for these consortia as they would clearly align with their organizational missions, while also taking advantage of (and helping to grow) their wellestablished networks of connections with both researchers and practitioners. Such infrastructure would also help forge connections between schools, out-of-school program owners, and researchers.

Such consortia could take better advantage of the strengths participating individuals and organizations have to offer. For example, programs that lack a research team would gain access to researchers, while researchers who don't work for programs can provide their expertise (and gain access to data). Additionally, consortia could also help remove concerns about programs only self-evaluating (c.f., Callahan \& Moon, 2007) through the addition of agreed upon research 
procedures. Such additions would bolster the rigor and trustworthiness of research findings. This approach could scale up established interventions. For example, interventions that have shown effectiveness in one setting or on a small scale, such as online mentoring (e.g., Stoeger, Hopp, \& Ziegler, 2017) could be implemented and tested on a much larger scale or in multiple contexts.

\section{Collaborative Analysis}

In addition to collaboration across different studies, researchers can also collaborate to analyze a single set of data through collaborative analysis. This can be valuable in demonstrating the vast number of potential routes researchers can pursue in a single investigation, known as the garden of forking paths (Gelman \& Loken, 2013). Such paths include which datapoints to omit, what analyses to run, what moderators to include, etc. Rather than burying these paths, collaborative analysis highlight the effects these decisions can have on research outcomes by creating a multiverse of research results that illustrate the heterogeneity of effects that could be found in a single dataset that would otherwise be masked. A famous example of collaborative analysis is when 29 research teams analyzed the same data to assess whether soccer players' skin tone was associated with the rate at which they were given red cards (Silberzahn et al., 2018). This is an example of how assessing a real-world concern (i.e., the extent to which racism played a role in penalization) could be operationalized in research that has clear connections to the gifted education world where equity concerns have long existed. Despite using the same data to answer the same research question with the same outcome, research teams made numerous different analytic choices leading to varied results (20 were statistically significant and 9 were null, and effect sizes ranged from moderately large to almost zero). This example illustrates how seemingly small choices along the path to research findings can greatly impact those findings, potentially biasing the results. A collaborative team distributes analytic work and can show 
whether variation in analytic choices leads to different results. We believe such application will lead to greater accuracy and credibility of the findings as well as better understanding of what gifted programs do (or do not) accomplish.

Collaborative analysis could be used in gifted education to assess whether various identification practices yield equitable identification rates, whether various services are associated with academic growth, or any number of other ways. Using multiple analysts is particularly helpful when data are difficult to collect, complex, and amenable to many analytic approaches, such as datasets that include repeated measures of students nested within different classes and programs, longitudinal datasets tracking developmental outcomes, or datasets with multiple student assessments or data types (e.g., text, audio, video, etc.). All of these are common in gifted program evaluation research and collaborative analysis would help ensure a given finding is robust across analytic decisions and not simply one of many possible results.

\section{Preregistered Adversarial Collaborations}

By definition, collaborative work requires working together. But working together can be difficult when collaborators hold differing beliefs. Rather than allow such disagreement to curtail collaboration, we believe that it should catalyze it. One way to help resolve disagreements through collaborative work is preregistered adversarial collaboration (PAC). In PACs, the primary goal is for groups who disagree to come together to determine what data could be collected that would convince them to change their position. It is important to note that PACs are only appropriate when individuals are able to state a situation in which their beliefs could be changed. If nothing could change their beliefs, the disagreement extends beyond the sphere of influence of empirical data. Obviously, conducting PACs requires not just coordination but also political savvy to negotiate what can often be decades long disagreements. But resolving such 
disagreements could greatly benefit the field by helping stakeholders come together with a more unified data-based agreement. In psychology, several teams have developed protocols and checklists that help preregistered adversarial collaborations succeed (Kerr, Ao, Hogg, \& Zhang, 2018; Latham, Erez, \& Locke, 1988; Matzke et al, 2015; Mellers, Hertwig, \& Kahneman, 2001; Neir \& Campbell, 2013).

PACs can help program owners avoid the familial level of unconditional positive regard that they may hold for their programs that can lead to problems of confirmation bias. Moreover, they could resolve disagreements. The smallness of the giftedness research field often facilitates shying away from seeing others as "adversaries", but polarizing beliefs exist. For example, some claim that gifted students are likely to be twice-exceptional despite the fact that there have been no epidemiological studies assessing such rates (c.f. Pfeiffer \& Foley-Nicpon, 2018). There is also disagreement about what constitutes appropriate measures of program success or even the purpose of gifted education (e.g., Dai \& Chen, 2013). Is it participant satisfaction? Future economic and/or creative successes? Self-report of self-actualization? We believe that PACs addressing these questions could lead toward a more unified field that provides stakeholders with consistent and accurate information about various learning opportunities.

\section{Discussion}

There are a variety of opportunities to identify and serve gifted and talented students. If the field of gifted education is serious about fostering the growth and development of advanced learners, we believe that it should take action to provide precise and accurate information to stakeholders. We believe that large scale collaborative research practices hold the potential to provide substantive value to assessing learning contexts. Many of them seem especially wellsuited to programs in gifted education because such programs are often somewhat similar to 
other programs, geographically isolated, and/or relatively small in the number of students they serve. If collaborative research approaches were implemented in gifted education contexts, we believe they could help A. provide more precise and accurate information about expected effects of participation; B. reveal heterogeneity of expected effects across contexts; C. help resolve disagreements within and beyond the field; and D. evolve to exploring new questions with greater confidence in previous findings.

Of course, all of these are testable hypotheses. To test them, the field would need to implement these practices and assess the effects. Large-scale collaboration would require an open presentation of assumptions, practices, and agreed upon strengths/weakness of different programs. We believe this alone would generate value to stakeholders as they make decisions about where to invest their resources. Additionally, fostering large-scale collaborative research would improve not only research, but also the accuracy of promises that programs make to the families that they serve. Individual programs would gain access to more and better information on the effects of their specific programs and how those effects are similar to/different from other programs.

Which form of large-scale collaboration would be most useful will largely depend on the several factors including, the question being addressed, existing knowledge of the question, agreement levels about that knowledge, and resources available.

\section{The Future of Collaborative Research in Gifted Education}

Although we believe there is great promise in collaborative research, there we expect there will also be barriers to implementation and limitations to benefits. There will assuredly be difficulties in implementation, not the least of which will be recruiting collaborators. There may also be areas of relative strength and weakness within the field for utility and ease of 
implementation of various collaborative strategies that will be discovered upon implementation.

But these are limitations of any new practice and not unique to large-scale collaborative research.

Substantial financial investments are at stake for many stakeholders. Programs may be unable to show desired outcomes. However, increased openness will lead to better understanding of what programs work for what kinds of participants and how these programs meet specific needs. Collaborations will also help demonstrate to programs where they need to improve to better align their actions with their aspirations. We believe that few program directors would choose not to have this knowledge. Yet, there are social and professional risks of making this information public and there is an inherent asymmetry in these risks, with programs taking all the risk, while the public gains information. Public awareness of program evaluation can come with social and financial risk creating a perverse incentive for programs to selectively filter what information is shared. Similar struggles have been found in research as open practices become more prevalent (e.g., Abele-Brehm, Gollwitzer, Steinberg, \& Schonbrodt, 2019). However, anecdotally, researchers whose work has been called into question who have admitted their errors and worked to correct them have often been well-received and serve as role models for others (e.g., psychologist Will Gervais). On the other hand, researchers who have stonewalled and denied error have experienced some severe consequences as extreme as losing tenured positions (e.g., Brian Wansink). In both cases (programs and researchers), uncovering and sharing strengths and areas needing improvement are not about vilifying individuals (or programs); it's about identifying effective practices and areas where additional work is needed.

To be successful, large-scale collaborative research initiatives should address topics that are of high interest to the field. But because these initiatives are new, early adopters should begin with manageable projects. Which projects are best suited for these types of collaborations is 
debatable. Beginning with relatively straightforward opportunities will allow all involved can learn the process before advancing to more complicated topics.

\section{Conclusion}

Given the variety of offerings for gifted and talented students, the gifted education community needs credible research and evaluation to provide precise and accurate information to stakeholders. Because gifted programs often exist in resource-limited environments and have few students, they could benefit from greater implementation of collaborative research. As researchers, we believe it is of utmost priority to avoid being purveyors of inaccuracy and to provide clarity of how confident (or not) stakeholders should be in relevant research evidence and in the program offerings. But it is easy to be wrong. We believe that a sign of a mature research field is one that has a clear sense of what has empirical support and how to uncover things that do not. We hope that gifted and talented research is at that stage of development so that we can begin collaborations that will bring more credibility to research findings and better meet the needs of students. 


\section{References}

Aad, G., Abajyan, T., Abbott, B., Abdallah, J., Abdel Khalek, S., Abdelalim, A. A., ... Abolins, M. (2012). Observation of a new particle in the search for the Standard Model Higgs boson with the ATLAS detector at the LHC. Physics Letters B, 716, 1-29. doi:10.1016/j.physletb.2012.08.020

Abbott, B. P., Abbott, R., Abbott, T. D., Acernese, F., Ackley, K., Adams, C., ... \& Affeldt, C. (2017). GW170817: Observation of gravitational waves from a binary neutron star inspiral. Physical Review Letters, 119, 161101. https://doi.org/10.1103/PhysRevLett.119.161101

Abele-Brehm, A. E., Gollwitzer, M., Steinberg, U., \& Schonbrodt, F. D. (2019). Attitudes toward open science and public data sharing. Social Psychology, 50, 252-260. http://dx.doi.org/10.1027/1864-9335/a000384

Adelson, J. L., Barton, E., Bradshaw, C., Bryant, B., Bryant, D., Cook, B. G., ... Troia, G. A. (2019). A roadmap for transparent research in special education and related disciplines. Retrieved from: https://doi.org/10.31219/osf.io/sqfy3

Adelson, J. L \& Matthews, M. S. (2019). Gifted Child Quarterly's commitment to transparency, openness, and research improvement. Gifted Child Quarterly, 62, 83-85. doi: $10.1177 / 0016986218824675$

Anderson, S. F., Kelley, K., \& Maxwell, S. E. (2017). Sample-size planning for more accurate statistical power: A method adjusting sample effect sizes for publication bias and uncertainty. Psychological Science, 28, 1547-1562. doi:10.1177/0956797617723724 
Callahan, C. M. \& Moon, T. R. (2007). Sorting the wheat from the chaff: What makes good evidence of effectiveness in the literature in gifted education? Gifted Child Quarterly, 51, 305-319.

Chatrchyan, S., Khachatryan, V., Sirunyan, A. M., Tumasyan, A., Adam, W., Aguilo, E., ... Fabjan, C. (2012). Observation of a new boson at a mass of $125 \mathrm{GeV}$ with the CMS experiment at the LHC. Physics Letters B, 716, 30-61.

doi:10.1016/j.physletb.2012.08.021Cook, B. G., Lloyd, J. W., Mellor, D., Nosek, B. A., \& Therrien, W. J. (2018). Promoting open science to increase the trustworthiness of evidence in special education. Exceptional Children, 85, 104-118.

doi:10.1177/0014402918793138

Dai, D. Y. \& Chen, F. (2013). Three paradigms of gifted education: In search of conceptual clarity in research and practice. Gifted Child Quarterly, 57, 151-168. DOI: $10.1177 / 0016986213490020$

de Vries Y. A., Roest, A.M., de Jonge, P., Cuijpers, P., Munafò, M. R., \& Bastiaansen, J.A. (2018). The cumulative effect of reporting and citation biases on the apparent efficacy of treatments: The case of depression. Psychological Medicine, 1-3. https://doi.org/ $10.1017 / \mathrm{S} 0033291718001873$

Gentry, M., Paul, K. A., McIntosh, J., Fugate, C. M., \& Jen, E. (2014). Total school cluster grouping: A comprehensive, research-based plan for raising student achievement and improving teacher practices, 2nd Edition. Waco, TX: Prufrock.

Ioannidis, J. P. A. (2005). Why most published research findings are false. PLoS Medicine, 2, 696-701. doi:10.1371/journal.pmed.0020124 
Ioannidis, J. P. A. (2014). How to make published research true. PLoS Medicine, 11, e1001747. doi:10.1371/journal.pmed.1001747

John, L. K., Loewenstein, G., \& Prelec, D. (2012). Measuring the prevalence of questionable research practices with incentives for truth telling. Psychological Science, 23, 524-532. doi:10.1177/0956797611430953

Kerr, N. L., Ao, X., Hogg, M. A., \& Zhang, J. (2018). Addressing replicability concerns via adversarial collaboration: Discovering hidden moderators of the minimal intergroup discrimination effect. Journal of Experimental Social Psychology, 78, 66-76. http://dx.doi.org/10.1016/j.jesp.2018.05.001

Kornrich, S. (2016). Inequalities in parental spending on young children: 1972 to 2010. AERA Open, 2(2), 1-12. doi:10.1177/2332858416644180

Latham, G. P. Erez, M., \& Locke, E. A. (1988). Resolving scientific disputes by the joint design of crucial experiments by the antagonists: Application to the Erez-Latham dispute regarding participation in goal setting. Journal of Applied Psychology, 73, 753 - 772. http://dx.doi.org/10.1037/0021-9010.73.4.753

Makel, M. C. \& Plucker, J. A. (2014). Facts are more important than novelty: Replication in the education sciences. Educational Researcher, 43, 304-316. doi: $10.3102 / 0013189 X 14545513$

Makel, M. C., Smith, K. N., McBee, M., Peters, S. J., \& Miller, E. M. (2019). Open science 2.0: Large-scale collaborative education research. Retrieved from psyarxiv.com/ypmjg Matzke, D., Nieuwenhuis, S., van Rijn, H., Slagter, H. A., van der Molen, M. W., \& Wagenmakers, E-J. (2015). The effects of horizontal eye movement on free recall: A 
preregistered adversarial collaboration. Journal of Experimental Psychology-General, 144, e1 - e15. https://doi.org/10.1037/xge0000038

Maxwell, S. E., Kelley, K., \& Rausch, J. (2008). Sample size planning for statistical power and accuracy in parameter estimation. Annual Review of Psychology, 59, 537-563. doi:10.1146/annurev.psych.59.103006.093735

McBee, M. T. \& Makel, M. C. (2019). The quantitative implications of definitions of giftedness. AERA Open. doi:10.1177/2332858419831007

McBee, M. T., Makel, M. C., Peters, S. J., \& Matthew, M. S. (2018). A call for open science in giftedness research. Gifted Child Quarterly, 62, 374-388. doi: $10.1177 / 0016986218784178$

Mellers, B., Hertwig, R., \& Kahneman, D. (2001). Do frequency representations eliminate conjunction effects? An exercise in adversarial collaboration. Psychological Science, 12, 269 - 275. http://dx.doi.org/10.1111/1467-9280.00350

Merton, R. K. (1973). The sociology of science: Theoretical and empirical investigations. Chicago, IL: University of Chicago Press. (Original work published 1942)

Moshontz, H., Campbell, L., Ebersole, C. R., IJzerman, H., Urry, H. L., Forscher, P. S., ... Chartier, C. R. (2018). The psychological science accelerator: Advancing psychology through a distributed collaborative network. Advances in Methods and Practices in Psychological Science, 1, 501-515. doi:10.1177/2515245918797607

Neir, J. A. \& Campbell, S. D. (2013). Two outsiders' view on feminism and evolutionary psychology: An opportune time for adversarial collaboration. Sex Roles, 69, 503-506. https://doi.org/10.1007/s11199-012-0154-2 
Open Science Collaboration. (2015). Estimating the reproducibility of psychological science. Science, 349(6251), aac4716-aac4716. doi:10.1126/science.aac4716

Rogers, K. B. (2015). The academic, socialization, and psychological effects of acceleration: Research synthesis. In S. G. Assouline, N. Colangelo, \& J. VanTassel-Baska (Eds.). A Nation Empowered. (pp. 19-29). Iowa City, IA: The Connie Belin \& Jacqueline N. Blank International Center for Gifted Education and Talent Development.

Schneider, D., Hastings, O. P., LaBriola, J. (2018). Income inequality and class divides in parental investments. American Sociological Review, 83, 475-507. doi:10.1177/0003122418772034

Silberzahn, R., Uhlmann, E. L., Martin, D. P., Anselmi, P., Aust, F., Awtrey, E., ... \& Carlsson, R. (2018). Many analysts, one data set: Making transparent how variations in analytic choices affect results. Advances in Methods and Practices in Psychological Science, 1, 337-356. https://doi.org/10.1177\%2F2515245917747646

Steenbergen-Hu, S., Makel, M. C., Olszewski-Kubilius, P. (2016). What one hundred years of research says about the effects of ability grouping and acceleration on K-12 students' academic achievement: Findings from two second-order meta-analyses. Review of Educational Research, 86, 849-899. doi:10.3102/0034654316675417

Stoeger, H., Hopp, M., \& Ziegler, A. (2017). Online mentoring as an extracurricular measure to encourage talented girls in STEM (science, technology, engineering, and mathematics): An empirical study of one-on-one versus group mentoring. Gifted Child Quarterly, 61, 239-249. doi: 10.1177/0016986217702215 
Uhlmann, E. L., Chartier, C. R., Ebersole, C. R., Errington, T. M., Kidwell, M., Lai, C. K., ... Nosek, B. A. (2018, August 13). Scientific utopia III: Crowdsourcing science. Retrieved from https://doi.org/10.31234/osf.io/vg649

Winebrenner, S., \& Brulles, D. (2008). The cluster grouping handbook: A schoolwide model: How to challenge gifted students and improve achievement for all. Minneapolis, MN: Free Spirit Publishing.

Wolf, R., Morrison, J., Slavin, R., Risman, K. (2019). Do developer-commissioned evaluations inflate effect sizes? Presented at the annual meeting of SREE in Washington DC.

Retrieved from: https://hechingerreport.org/wp-content/uploads/2019/03/developerabstract.pdf

van der Zee, T., \& Reich, J. (2018). Open education science. AERA Open, 4(3), doi: 2332858418787466.

Vazire, S. (2018). Implications of the credibility revolution for productivity, creativity, and progress. Perspectives on Psychological Science, 13, 411-417. doi:10.1177/1745691618751884 